\title{
Epidemiología de la depresión en el adulto mayor
}

\author{
Epidemiology of depression in the elderly \\ Diego Calderón M. 1,a; 2, b,c

\section{RESUMEN}

La depresión y la demencia son las enfermedades neuro-psiquiátricas de mayor prevalencia en el adulto mayor. En la depresión de este tipo de población influyen factores biológicos y sociales que son modificables. Se realizó una revisión de la bibliografía desde el año 2000 con el objetivo de contribuir al conocimiento de la epidemiología de la depresión del adulto mayor. Se encontró que la prevalencia varía según la localidad, país y también según la herramienta utilizada para el diagnóstico. De la exposición de los factores de riesgo se desprende que la familia, la sociedad y el estado deben emprender acciones específicas para paliar especialmente los factores modificables como el apoyo social y la mejora de la calidad de vida de los ancianos.

PALABRAS CLAVE: Depresión, prevalencia, factores de riesgo, calidad de vida. (Fuente: DeCS BIREME).

\section{SUMMARY}

Depression and dementia are the most prevalent neuro-psychiatric diseases in the elderly. Depression on this type of population is influenced by biological and social factors that are modifiable. A review of the literature was carried out since the year 2000 with the goal of contributing to the knowledge of the epidemiology of depression in the elderly. It was found that the prevalence varies according to the locality, country and also according to the tool used for the diagnosis. It is clear from the exposure of risk factors that the family, society and government should take specific actions to alleviate the modifiable factors such as social care and the improvement of quality of life of the elderly.

KEYWORDS: Depression, prevalence, risk factors, quality of life. (Source: MeSH NLM).

\section{INTRODUCCIÓN}

El aumento de la esperanza de vida a nivel mundial debe ir de la mano de una mejor calidad de vida en esos años. Actualmente, la esperanza de vida es de 72,7 años en hombres y 78,1 años en mujeres (1). En Ecuador se estima que $7 \%$ de la población es mayor de 65 años, el dato concuerda con la estadística mundial (2).
El adulto mayor tiene mayor morbilidad y padece especialmente enfermedades crónicas tanto físicas como mentales, las cuales aumentan progresivamente la dependencia (3). Demencia y depresión tienen alta prevalencia en el adulto mayor, en Ecuador la prevalencia fue de 39\% según la encuesta SABE (Salud, Bienestar y Envejecimiento) (4). La depresión en esta población tiene características especiales pues

Hospital Carlos Andrade Marín. Quito, Ecuador.

Facultad de Medicina, Pontificia Universidad Católica del Ecuador. Quito, Ecuador.

Médico; ${ }^{\mathrm{b}}$ Profesor; ${ }^{\mathrm{c}}$ Magíster en Gestión de Desarrollo Social. Profesor 
las personas mayores de 65 años tienen factores propios como las enfermedades que padecen, el entorno en que viven, situación social e incluso algunos factores demográficos como trabajo, estado civil, entre otros. La soledad predispone a depresión en mujeres y hombres (5). Un estudio en Perú encontró asociación de depresión con problemas físicos e incapacidad (6).

La depresión es un proceso multifactorial y se ha demostrado asociación con la falta de apoyo de la red social. En Tailandia sólo 38\% de personas mayores de 80 años con depresión, tenía buena red social (7).

La presente revisión tuvo como objetivo contribuir al conocimiento de la epidemiología de la depresión en el adulto mayor y de los factores asociados, para ello se realizó una búsqueda bibliográfica en bases como MEDLINE, PLoS, Scielo, desde el año 2000.

\section{Incidencia y prevalencia}

Se estima que la incidencia de casos nuevos por año es de aproximadamente 15\% (8). Un estudio longitudinal en Beijing encontró una incidencia acumulada a cuatro años de $10,58 \%$ en una muestra de 2506 ancianos de las zonas urbana y rural (9). Sigström et al., en Suecia, reportaron una incidencia de $16,4 \%$ a cinco años en una cohorte de personas de 70 años (10); cabe destacar que la depresión mayor tuvo un incremento importante de la incidencia en personas con antecedentes de algún grado de depresión a lo largo de su vida en comparación con personas sin antecedente $(10,3 \%$ vs $1,8 \%)$.
La prevalencia de depresión en el adulto mayor varía según la población y el desarrollo económico de los países (11). El estudio Eurodep, utilizando un cuestionario para ese continente, reportó 8,8\% en Islandia y $23,6 \%$ en Alemania (12). En Suecia se reporta una prevalencia de 12 a 14\% (13) y en Taiwán $21,2 \%$ (14).

Un estudio reciente en población de adultos mayores ( $\mathrm{n}=17$ 000) utilizando los criterios CIE-10 reportó prevalencias de 2,3\% en Puerto Rico, 13.8\% en República Dominicana, 4,9\% en Cuba, 0,3\% en China urbana, $0,7 \%$ en China rural, 3,9\% India urbana, $12,6 \%$ India rural y $0,5 \%$ en Nigeria (15). Cabe recalcar que las cifras son menores cuando se utilizan instrumentos diagnósticos, como los del CIE 10 o los de DSM-V en comparación con instrumentos de tamizaje, como la escala de Yesavage o el Euro-dep (Tabla 1).

En Latinoamérica los datos de prevalencia en el adulto mayor también son variados. En México, varía entre $26 \%$ y $66 \%$; un estudio en población marginal reportó depresión de 60\% (16). En Perú, se reporta $15,97 \%$ de depresión en pacientes hospitalizados (17) y $9,8 \%$ en la comunidad (18). En Colombia, un estudio en pacientes ambulatorios encontró depresión en $11,5 \%$ de mujeres y $4 \%$ de hombres (19); otro estudio multicéntrico encontró $47 \%$ de depresión en pacientes hospitalizados (20) y la encuesta nacional de salud reportó de 49 a $60 \%$ de depresión en adultos mayores (21). En Chile, la prevalencia de depresión se ha reportado entre $7,6 \%$ y $16,3 \%$ (22).

Tabla 1. Prevalencia de depresión con CIE-10 y Euro-dep.

\begin{tabular}{|c|c|c|c|c|c|c|}
\hline \multirow{2}{*}{ País } & \multicolumn{3}{|c|}{ CIE-10 (\%) } & \multicolumn{3}{|c|}{ Euro-dep (\%) } \\
\hline & Hombres & Mujeres & Total & Hombres & Mujeres & Total \\
\hline Cuba & $2,6(1,6-3,6)$ & $5,2(3,3-7,2)$ & $4,9(4,1-5,7)$ & $9,5(7,7-11,3)$ & $20,3(18,4-22,1)$ & $16,5(15,1-17,9)$ \\
\hline $\begin{array}{l}\text { República } \\
\text { Dominicana }\end{array}$ & $11,1(8,8-13,5)$ & $15,2(13,3-7,2)$ & $13,8(12,3-15,3)$ & $19,6(16,6-22,5)$ & $30,6(28,1-33,2)$ & $26,8(24,8-28,8)$ \\
\hline Puerto Rico & $1,2(0,4-2,2)$ & $2,8(1,9-3,7)$ & $2,3(1,7-3,0)$ & $6,3(4,4-8,2)$ & $12,6(10,8-14,5)$ & $10,6(9,2-12,0)$ \\
\hline China urbano & No casos & $0,5(0,0-1,0)$ & $0,3(0,0-0,6)$ & $1,9(0,7-3,1)$ & $3,0(1,7-4,3)$ & $2,5(1,6-3,4)$ \\
\hline China rural & $0,9(0,0-1,8)$ & $0,5(0,0-1,1)$ & $0,7(0,2-1,2)$ & $1,4(0,3-2,5)$ & $0,7(0,0-1,5)$ & $1,0(0,3-1,7)$ \\
\hline India urbano & $4,3(2,4-6,2)$ & $3,7(2,1-5,2)$ & $3,9(2,7-5,1)$ & $20,9(17,0-24,8)$ & $34,6(30,6-38,5)$ & $28,6(25,7-31,5)$ \\
\hline India rural & $13,2(10,1-16,3)$ & $12,1(9,4-14,8)$ & $12,6(10,5-14,7)$ & $36,7(32,2-41,2)$ & $40,2(35,9-44,4)$ & $38,6(35,3-41,9)$ \\
\hline Nigeria & $0,5(0,0-1,3)$ & $0,6(0,0-1,2)$ & $0,5(0,1-1,0)$ & $18,8(14,9-22,7)$ & $22,7(19,5-26,0)$ & $21,1(18,8-23,5)$ \\
\hline
\end{tabular}

Fuente: Guerra M, Prina AM, Ferri CP, et al. (12) 
En Ecuador, de acuerdo con proyecciones del Instituto Ecuatoriano de Estadísticas y censos (INEC), viven 1229089 adultos mayores con una proporción de mujeres de 53\% y un índice de feminidad de 115 (23). En Ecuador se han realizado pocos estudios, la mayor parte de ellos en adultos mayores hospitalizados o residentes de asilos. Un estudio en pacientes residentes en asilo encontró depresión en $70 \%$ (24). Otro estudio en una pequeña población rural con alta proporción de emigrantes reportó 93\% de depresión (25), datos elevados que no se pueden inferir a la población general. No se encontraron estudios en la comunidad ni en pacientes afiliados a la seguridad social o jubilados, excepto la prevalencia de depresión de 39\% reportada en la encuesta SABE (4). El proyecto Atahualpa, estudio realizado en una población marginal de la Sierra, en 280 personas mayores de 60 años, encontró una prevalencia de depresión de $12 \%$ y una asociación importante con deterioro cognitivo (26).

\section{Factores de riesgo}

En la depresión del adulto mayor influyen varios factores, tanto genéticos, biológicos, psicológicos y ambientales (27). Varios factores anatómicos tratan de explicar la depresión, entre ellos el tamaño diferente de hipocampo, parahipocampo, amígdala y corteza prefrontal $(28,29)$. También se ha postulado, a neuronas con alteración de su metabolismo y tamaño, acción de las monoaminas y otros neurotransmisores, vulnerabilidad genética, estrés, e inflamación (30). La etiología vascular parece relacionarse con la depresión tardía (31). Por mucho tiempo, se ha estudiado el papel de la serotonina y se considera que en la depresión la alteración de la serotonina afecta a personas vulnerables (32).

A continuación, se analizan los factores de riesgo más importantes:

\section{a. Demográficos}

La gran mayoría de estudios reporta que la proporción de depresión es más alta en mujeres que en hombres. Las viudas, ancianos aislados, pobres, institucionalizados y sometidos a estrés presentan depresión más frecuentemente (33). La prevalencia es mayor en el área rural que en la urbana (14). Un estudio en Arabia, encontró relación entre depresión y desempleo, divorcio, viudez, soledad y bajo nivel de instrucción ( $\mathrm{n}=7970)$ (34).

\section{b. Biológicos}

\section{Enfermedad orgánica}

Varias enfermedades han sido relacionadas con la depresión en el adulto mayor, quienes, por características propias, tienen mayor comorbilidad. Un estudio reciente de comorbilidades en adultos menores y mayores de 65 años realizado en los Estados Unidos de Norteamérica (USA) en 201711 personas, concluyó que para disminuir la carga de enfermedades crónicas, los adultos menores de 65 años con enfermedades crónicas deben recibir el tratamiento que necesitan para reducir la probabilidad de desarrollar más enfermedades crónicas a medida que envejecen, el objetivo final es mejorar el estado de salud y reducir los costos de atención médica para padecimientos crónicos en adultos mayores (35). Se ha demostrado asociación entre enfermedad cardiovascular y depresión, en muchos casos la depresión se presenta antes que el trastorno cardiovascular (36).

Se han encontrado patrones de morbilidad más frecuentes en adultos mayores, por ejemplo, enfermedades cardio-metabólicasy depresión, ansiedad y depresión, y dolor-desórdenes neuropsiquiátricos y depresión (37). Diabetes y cardiopatías han sido relacionadas con la depresión; el estudio ELSA (English Longitudinal Study of Ageing), realizado en 4454 pacientes diabéticos adultos mayores, demostró asociación entre depresión-diabetes y diabetestrastornos cardiovasculares (38).

Se observa una alta prevalencia de depresión en pacientes con enfermedades cerebrovasculares. Camus et al., hacen referencia al término "Depresión vascular" para describir los trastornos depresivos consecutivos a enfermedad cerebrovascular (39). Un estudio de cohorte retrospectivo realizado en Taiwan en 9991 pacientes con depresión y 39964 controles, sugiere que la depresión es un factor de riesgo independiente de la demencia vascular (40).

\section{Deterioro funcional}

La limitación funcional en los adultos mayores está relacionada con depresión y sentimientos de inutilidad. Un estudio en USA encontró asociación entre síntomas depresivos y alteraciones en la marcha (41). En México, un estudio en pacientes hospitalizados encontró que $62 \%$ era dependiente y que existía relación directa entre depresión y disfunción (42). En Turquía, un estudio transversal sobre una población 
de 19853 personas mayores de 60 años, reportó un aumento de la depresión en relación a una disminución de las actividades de la vida diaria (Escala de Katz) y un aumento ante la disminución de la calidad de vida (43).

La fragilidad en adultos mayores se entiende como pérdida de energía, masa, fuerza muscular, reserva fisiológica disminuida e intolerancia al ejercicio (44); depresión y fragilidad coexisten en $10 \%$ de adultos mayores (45).

\section{Deterioro cognitivo}

La depresión y su relación con el deterioro cognitivo tiene amplia evidencia científica. La depresión puede ser un signo de demencia incipiente $\mathrm{y}$ algunos pacientes con depresión permanecen sin síntomas mientras no tienen demencia (46).

La relación causa-efecto entre depresión y demencia es muchas veces difícil de establecer, además, se ha observado que la depresión en el adulto mayor, puede preceder a la demencia (47). Un estudio realizado en 195 adultos mayores con deterioro cognitivo, encontró más relación entre depresión y Alzheimer que con otros tipos de demencia (48). Una revisión sobre la relación entre cognición y depresión encontró que la dificultad de integración social en la depresión mayor puede deberse, en parte, a la dificultad de interpretar los estímulos en la demencia (49).

Algunos estudios han reportado que la demencia puede influir en la respuesta al tratamiento de la depresión; sin embargo, la evidencia no es clara, un estudio realizado en 302 adultos mayores encontró que la coexistencia de deterioro cognitivo dificulta el tratamiento de la depresión en estos pacientes y que la valoración de la cognición debe realizarse en todos los pacientes con depresión (50), en Australia en cambio, un estudio prospectivo encontró similar proporción de uso de antidepresivos en ancianos con y sin demencia (51).

\section{Insomnio}

Como la demencia, el insomnio está relacionado con la depresión, pero puede ser un factor o un efecto. El estudio multicéntrico IMPACT, en 1801 adultos mayores, encontró que los pacientes con insomnio tenían mayor probabilidad de tener depresión y mayor persistencia de la enfermedad (52). Otro estudio, en USA, reportó que el insomnio en un año aumenta la prevalencia de depresión al siguiente año, recomendando incluso la detección de alteraciones del sueño para un mejor tratamiento de la depresión (53). Un estudio en tres ciudades de Francia en 3824 adultos mayores concluyó que los síntomas del insomnio y el uso de medicación aumentan el riesgo de depresión (54).

\section{c. Sociales}

El aislamiento es un factor importante asociado con la depresión en el adulto mayor ya que puede por sí mismo provocar depresión o agravarla. Puede además influir de manera negativa en el estado de salud y la calidad de vida (55). Nazemi et a., en Irán, reportaron $90 \%$ de depresión en adultos mayores asilados (56). En China se encontró una prevalencia de $36,94 \%$ en ancianos abandonados de zonas rurales (57).

La depresión es más frecuente en situación de riesgo social y con baja condición socio económica (58). Siversten et al., en una revisión reciente encontraron que la depresión es más frecuente y más grave en adultos mayores con menor calidad de vida (59). Un estudio prospectivo a 6 años con 1991 adultos mayores encontró fuerte asociación entre dolor generalizado y depresión, con reducción de la participación social (60). También se ha demostrado que la depresión mayor, bajos ingresos económicos, enfermedades crónicas y dependencia influyen en la ideación suicida (61). En Brasil, un estudio transversal en 1012 personas mayores de 60 años, encontró menor frecuencia de depresión en personas con indicadores de envejecimiento activo, como tener amigos, trabajo y hábito de leer (62).

\section{d. Económicos}

Es evidente que la situación económica influye en el estado de ánimo de las personas, es más probable que la persona con bajos ingresos económicos se encuentre triste, esto se aplica a todas las edades. Una encuesta a adultos mayores en China y Rusia atribuyó el menor nivel de depresión en China a mayores niveles de seguridad económica y cohesión social, es decir, a los efectos de los recursos económicos y sociales sobre la depresión (63).

Las crisis financieras en los países influyen directamente sobre la situación económica de sus habitantes, el estudio MEDIS analizó como la crisis financiera en el Mediterráneo afectó los determinantes de salud de los adultos mayores; en efecto, la 
crisis aumentó la depresión, el aislamiento social, tabaquismo, y afectó la calidad de dieta y disminuyó la actividad física (64).

Las circunstancias económicas pueden ser importantes para determinar la satisfacción y el estado de ánimo de la vida, no sólo por la situación objetiva, sino también por su interpretación subjetiva. Lo que para una persona puede ser una quiebra financiera para otra persona puede no serlo, por tanto, variables subjetivas como la autoestima de la salud, la situación económica y la satisfacción con la vida deben considerarse al abordar al paciente deprimido (65).

\section{Impacto}

La depresión del adulto mayor agrava las comorbilidades, aumenta las necesidades de atención de salud incluyendo la atención de hospitalización y emergencia, aumenta la discapacidad, deteriora la calidad de vida, aumenta el consumo de drogas y alcohol. Además, aumenta el riesgo de muerte especialmente en síndromes depresivos mayores, como lo demostraron Schoevers et al., en un seguimiento a 10 años de 3746 adultos mayores (66). Alentar a los individuos a iniciar, mantener y expandir el compromiso social en los últimos años de vida podría ayudar a mantener y mejorar la calidad de vida relacionada a la salud y disminuir los síntomas depresivos (67).

Los gastos de salud en adultos mayores con depresión se incrementan de manera importante por complicaciones en enfermedades crónicas como insuficiencia cardiaca y diabetes (68). El adulto mayor es usuario frecuente de los servicios de salud; en Ecuador, 7 de cada 10 adultos mayores acudieron a los servicios médicos para ser atendidos, $38 \%$ lo hicieron a un servicio público (69), las visitas al médico aumentan en pacientes deprimidos.

\section{a. Comorbilidad y mortalidad}

El control y tratamiento de la depresión en adultos mayores con enfermedades crónicas como la artritis es muy importante, en efecto, el tratamiento con antidepresivos y psicoterapia mejoró no solo la depresión sino además disminuyó el dolor, mejoró el estado funcional y la calidad de vida (70). En pacientes con diabetes y depresión, el tratamiento antidepresivo integral mejoró el estado afectivo y funcional especialmente en pacientes sin buen control de glicemia (71).
El cuidado de los adultos mayores con depresión y enfermedad cardiovascular debe incluir intervenciones sobre el estilo de vida y factores psicológicos. La prevención y tratamiento de la depresión debe ser parte del manejo integral de las enfermedades cardiovasculares en el adulto mayor (72).

El deterioro cognitivo y la depresión, enfermedades que frecuentemente están asociadas en el adulto mayor, parece aumentar la mortalidad por todas las causas $(73,74)$ La comorbilidad debe ser cuidadosamente evaluada, pues parece ser un índice independiente de mayor fragilidad, que acorta la esperanza de vida (75). Los síntomas neuro-psiquiátricos, entre ellos la depresión, obstaculizan el proceso de rehabilitación después de un accidente cerebrovascular, deterioran el nivel de vida, provocan institucionalización y agregan carga económica y emocional a la familia (76).

El riesgo de mortalidad a largo plazo asociado a depresión en hogares de ancianos, depende de su detección oportuna; mejor pronóstico tienen los residentes con depresión detectada. Es importante la búsqueda de síntomas depresivos en todo paciente institucionalizado (77).

\section{b. Trastornos psiquiátricos}

La depresión es un factor de riesgo para la demencia, pero no está claro si es causal. Un estudio longitudinal de 14 años en 4922 personas de entre 71 y 89 años, encontró asociación entre demencia y depresión, pero solamente los primeros 5 años; además, los hombres con historia de depresión tienen mayor riesgo de desarrollar demencia. Es probable que la depresión sea un marcador de demencia incipiente (78).

La depresión de inicio tardío se asocia más a menudo con deterioro cognitivo que la depresión temprana. Hashem et al., encontraron que la depresión de inicio tardío era más severa y afectaba mayormente la cognición en cuanto a memoria, fluidez verbal y habilidades visuoespaciales(79).

El uso de alcohol y drogas para aliviar el estado de ánimo es común en todas las personas, pero se asocia especialmente, con el incremento de la comorbilidad psiquiátrica, ansiedad y trastornos de personalidad (80); sin embargo, un estudio en USA reportó que la prevalencia de trastornos del humor, la ansiedad y el consumo de sustancias durante 12 meses y durante toda la vida fue menor para los adultos mayores de 65 años, que para los grupos de edad más jóvenes 
Tabla 2. Factores de riesgo y protectores de conducta suicida en adulto mayor.

\begin{tabular}{ll}
\hline \multicolumn{1}{c}{ Factores de riesgo } & \multicolumn{1}{c}{ Factores protectores } \\
\hline Conducta suicida previa y presencia de ideación suicida & Hábitos de vida saludables \\
Trastornos mentales y adicciones & Contacto con la familia y amigos \\
Trastornos y rasgos de personalidad & No consumo de alcohol \\
Enfermedad física & Mantenerse activo \\
Apoyo vital & $\begin{array}{l}\text { Práctica religiosa y sentimiento de tener un propósito } \\
\text { en la vida }\end{array}$ \\
Eventos vitales estresantes & Extroversión, apertura a la experiencia, responsabilidad \\
Déficit funcional & Percepción de autoeficacia. \\
& Orientación al futuro y espiritualidad \\
\hline
\end{tabular}

Fuente: SNS Guía de práctica clínica de prevención y tratamiento de la conducta suicida (84).

(81). Muchos adultos mayores reciben tratamiento farmacológico para trastornos neuro-psiquiátricos; las benzodiacepinas en combinación con antidepresivos se prescriben de manera inconsistente con las normas. Se debe tener en cuenta para la prescripción de antidepresivos los riesgos de esta práctica generalizada, caídas por ejemplo (82).

\section{c. Suicidio}

El suicidio es una importante causa de muerte en USA y las tasas aumentan en hombres blancos mayores de 75 años. Después de los 85 años, las tasas son 5 veces más altas que la población general y el consumo de alcohol eleva aún más el riesgo (83). El trastorno depresivo mayor recurrente fue un factor de riesgo muy fuerte para el suicidio, al igual que el trastorno por uso de sustancias. Un riesgo elevado también se asoció con trastorno depresivo menor, trastorno distímico, trastorno psicótico, trastorno depresivo mayor de un solo episodio y trastorno de ansiedad (84), en la tabla 2 se resumen factores de riesgo y protectores (85).

La atención primaria ofrece la oportunidad de llegar al adulto mayor que intenta suicidio. La investigación, detección y el tratamiento de la posibilidad de suicidio en atención primaria, puede ayudar a reducir las tasas de ideación y comportamiento suicida en los adultos mayores (86).

Las tasas de suicidios son más altas entre los ancianos, las estrategias para enfrentar el problema deben mejorar la resiliencia y el envejecimiento positivo, comprometer a familia y comunidad, utilizar medios de comunicación para llegar a los ancianos en riesgo y mejorar la educación de los médicos en el tema del suicidio en ancianos (87). La conducta suicida del adulto mayor tiene características propias, tiene menos intentos que los jóvenes, pero son más efectivos y da menos señales de aviso (88).

\section{CONCLUSIONES Y RECOMENDACIONES}

La depresión y la demencia son los trastornos neuro-psiquiátricos más frecuentes en el adulto mayor. La prevalencia varía según el desarrollo socio económico de los países; como es lógico suponer, es más frecuente en países en vías de desarrollo. La depresión en el adulto mayor tiene características propias y el impacto sobre la calidad de vida, las comorbilidades y la ideación suicida es evidente.

Es importante el conocimiento de los factores de riesgo de depresión para poder enfrentar el problema de manera más eficaz.

Es recomendable detectar la ideación suicida en el primer encuentro con el paciente geriátrico, en lo posible desde la atención primaria de salud. Se encuentran algunas escalas a disposición del médico.

La comunidad, los gobiernos y las instituciones internacionales deben tomar acciones para contrarrestar los factores de riesgo modificables y poner en práctica políticas sociales y de salud para mejorar la calidad de vida de los adultos mayores. 


\section{Correspondencia:}

Diego Calderón M.

Correo electrónico:dfcalderonm@yahoo.es

\section{REFERENCIAS BIBLIOGRÁFICAS}

1. Organización Mundial de la Salud. Estadísticas Mundiales: una mina de información sobre salud pública mundial. Ginebra: Organización Mundial de la Salud; 2014.

2. Instituto Nacional de Estadísticas y Censos. Resultados censo. Quito: Instituto Nacional de Estadísticas y Censos; 2010.

3. Rivera JM. Aspectos demográficos, sociosanitarios y biológicos del envejecimiento. Manual de Recomendaciones nutricionales en pacientes geriátricos. Madrid: Editores Médicos SA; 2004. p.25-30.

4. Instituto Nacional de Estadísticas y Censos. Salud, Bienestar y Envejecimiento. Lima: Instituto Nacional de Estadísticas y Censos;2009. p. 17-18.

5. Klung G, Lacruz M, Emeny R, et al. Aging without depression: a cross sectional study. Psychodyn Psychiatry. 2014; 42(1):5-22.

6. Paz V. Factores asociados al episodio depresivo en el adulto mayor de Lima metropolitana y Callao. Anales de Salud Mental. 2010; 26(1):30-38.

7. Aung M, Moolphate S, Aung T, et al. The social network index and its relation to later-life depression among the elderly aged $>80$ years in Northern Thailand. Clin Interv Aging. 2016;11:1067-74. doi: 10.2147/CIA.S108974

8. Sotelo-Alonso I, Rojas-Soto JE, Sánchez-Arenas C, Irigoyen-Coria A. Depresión en el adulto mayor: una perspectiva clínica y epidemiológica desde el primer nivel de atención. Arch Med Fam. 2012; 14(5):5-13.

9. Sun F, Tang Z, Liu FR. Study on the relationship between somatic health and incidence of depression in the elderly. Zhonghua Liu Xing Bing Xue Za Zhi. 2008; 29(2):121-4.

10. Sigström R, Waern M, Gudmundsson, P, Skoog I, Östling S. Depressive spectrum states in a population based cohort of 70 year olds followed over 9 years. Int J Geriatr Psychiatry. 2018; 33(8):1009-1159. doi: https://doi.org/10.1002/gps.4888

11. Beekman AT, Copeland JR, Prince MJ. Review of community prevalence of depression in later life. $\mathrm{Br} \mathrm{J}$ Psychiatry. 1999; 174:307-11.

12. Copelanf J, Beekman A, Braam A, Dewey M, Delespaul P, et al. Depression among older people in Europe: the EURODEP studies. World Psichiatry. 2004; 3(1):45-49.

13. Magnil M, Janmarker L, Gunnarsson R, Björkelund C. Course, risk factors and prognostics factors in elderly Primary Health Care. 2013; 31:20-25.

14. Chong MY, Tsang HY, Chen CS, et al. Community study of depression in old age in Taiwan: prevalence, fife events and socio-demographic correlates. $\mathrm{Br} \mathrm{J}$ Psychiatry. 2001; 178(1):29-35.

15. Guerra M, Prina AM, Ferri CP, , et al. A comparative cross-cultural study of the prevalence of late life depression in low and middle income countries. J Affect Disord. 2016; 190:362-8.

16. Durán T, Aguilar MR, Martínez ML, Rodríguez T, Gutiérrez G, Vásquez L. Depresión y función cognitiva de adultos mayores de una comunidad urbano marginal. Enfermería Universitaria. 2013; 10(2):36-42.

17. Varela L, Chávez H, Herrera A, Ortiz P, Chigne O. Valoración Geriátrica Integral en adultos mayores hospitalizados a nivel nacional. Diagnóstico. 2004; 43 (2):57-63.

18. Paz V. Factores asociados al episodio depresivo en el adulto mayor de Lima metropolitana y Callao. Anales de Salud Mental.2010; 26(1):30-38.

19. Ocampo J, Romero N, Saa H, Herrera J, Reyes C. Prevalencia de las prácticas religiosas, soporte social y síntomas depresivos en adultos mayores. Cali - Colombia 2001. Colombia Médica. 2006; 37(2):2630 .

20. Estrada A, Cardona D, Segura A, Ordóñez J, Osorio J. Síntomas depresivos en adultos mayores institucionalizados y factores asociados. Univ Psychol. 2013; 12(1):81-94.

21. Departamento Administrativo Nacional de Estadística, Ministro de Salud y Protección Social. Encuesta Nacional de Demografía y Salud: Colombia Informe 2011.Bogotá: Departamento Administrativo Nacional de Estadística, Ministro de Salud y Protección Social; 2012.

22. Mühlenbrock F, Gómez R, González M, Rojas A, Vargas L, Mühlenbrock C. Prevalencia de Depresión en Adultos Mayores de 60 años hospitalizados en el Servicio de Medicina Interna del Hospital Militar de Santiago. Rev chil Neuro-Psiquiat. 2011; 49(4):331-337.

23. Freire W. SABE Encuesta de Salud, Bienestar y Envejecimiento. Ecuador 2009, 2010 Presentación de resultados. Quito: Ministerio de Salud Pública del Ecuador; 2009.

24. Crespo J. Prevalencia de Depresión en adultos mayores de los asilos de los cantones Azogues, Cañar, Tambo y Deleg de la provincia de Cañar, año 2011. Tesis previa obtención de título de Médico. Cuenca, Ecuador: Universidad de Cuenca; 2011. 44 pp.

25. Andrade C. Nivel de Depresión en adultos mayores de 65 años y su impacto en el estado nutricional en la parroquia Asunción, cantón Girón, Azuay. 2011. Tesis Nutricionista dietista. Riobamba, Ecuador: Escuela Superior Politécnica de Chimborazo; 2011. 
$93 \mathrm{pp}$.

26. Del Brutto O, Mera R, Del Brutto V, et al. Influence of depression, anxiety and stress on cognitive performance in community-dwelling older adults living in rural Ecuador: results of the Atahualpa Project. Geriatr Gerontol Int. 2015;15(4):508-14

27. Mitjams M, Arias B. La genética de la depresión: ¿Qué información aportan las nuevas aproximaciones metodológicas? Actas Esp Psiquiatr. 2012; 40(2):7083.

28. Zhou H, Li R, Ma Z, Rossi S, Zhu X, Li J. Smaller gray matter volume of hippocampus/parahippocampus in elderly people with subthreshold depression: a cross-sectional study. BMC Psychiatry. 2016;16:219. doi: 10.1186/s12888-016-0928-0

29. Taki Y, Kinomura S, Awata S, et al. Male elderly subthreshold depression patients have smaller volume of medial part of prefrontal cortex and precentral gyrus compared with age-matched normal subjects: a voxel-based morphometry. J Affect Disord. 2005; 88(3):313-20.

30. Díaz B, González C. Actualidades en neurobiología de la depresión. Rev Latinoam Psiquiatria. 2012; 11(3):106-115.

31. Kales HC, Maixner DF, Mellow AM. Cerebrovascular disease and late-life depression. Am J Geriatr Psychiatry. 2005; 13(2):88-98.

32. Dell'Osso L, Carmassi C, Mucci F, Marazziti D. Depression, serotonin and tryptophan. Curr Pharm Des. 2016; 22(8):949-54.

33. Lykouras L, Rossetos G. Depression in the Elderly. Annals of General Psychiatry. 2008; 7(Suppl 1):S17 doi:10.1186/1744-859X-7-S1-S17

34. Sulaiman A, Al-Shammari, Al-Subaie A. Prevalence and corelates of Depression among Saudi elderly. Int. J. Geriat. Psychiatry. 1999; 14:739-749.

35. Adams ML.Differences Between Younger and Older US Adults With Multiple Chronic Conditions. Prev Chronic Dis. 2017;14:E76. doi: 10.5888/ pcd14.160613

36. O’Neil A, Williams E, Stevenson E, Oldenburg B, Berek M, Sanderson K. Co-morbid cardiovascular disease and depression: sequence of disease onset is linked to mental but not physical self-rated health. Results from a cross-sectional, populationbased study. Soc Psychiatry Psychiatr Epidemiol. 2012;47(7):1145-51.

37. Schäfer I, von Leitner E, Schön G, et al. Multimorbidity Patterns in the elderly: A new approach of disease clustering identifies complex interrelations between chronic conditions. PLoS ONE. 2016; 5(12):1-10. DOI: 10.1371/journal. pone. 0015941

38. Freitas C, Deschênes S, Au B, Smith K, Schmitz N. Risk of diabetes in older adults with co-occurring depressive symptoms and cardiometabolic abnormalities: Prospective analysis from the english longitudinal study of ageing. PLoS ONE. 2016; 11(5):1-16. DOI: 10.1371/journal.pone.0155741

39. Camus V, Kraehenbühl H, Preisig M, Büla C, Waeber G. Geriatric depression and vascular diseases: what are the links? Journal for Afective Disorders. 2004; 81(1):1-16

40. Lin WC, Hu LY, Tsai SJ, Yang AC, Shen. Depression and the risk of vascular dementia: a populationbased retrospective cohort study. Int $\mathrm{J}$ Geriatr Psychiatry. 2017;32(5):556-563.

41. Brandler T, Wang C, Oh-Park M, Holtzer R, Verghese J. Depressive Symptoms and Gait Dysfunction in the Elderly. Am J Geriatr Psychiatry. 2012; 20(5):425432.

42. Martínez J, Martínez V, Esquivel C, Velasco V. Prevalencia de Depresión y Factores de riesgo en el adulto mayor hospitalizado. Rev Med Inst Mex Seguro Soc. 2007; 45(1):21-28.

43. Unsar S, Dindar, Kurt S. Activities of daily living, quality of life, social support and depression levels of elderly individuals in Turkish society. J Pak Med Assoc. 2015; 65(6):642-6.

44. Buiques C, Padilla Sánchez C, Garrido JF, Navarro Martínez R, Ruiz-Roz V, Cauli O. The Relationship between Depression and Frailty síndrome: a systematic review. Aging Ment Health. 2015; 19(9):762-72.

45. Vaughan L, Corbin A, Goveas J. Depression and frailty in later life: A systematic review. Clin Interv Aging. 2015; 10:1947-1958.

46. Heser K, Bleckwenn M, Wiese B, Mamone S, RiedelHeller SG, Stein J, et al. Late-Life depressive symptoms and lifetime history of major depression: Cognitive deficits are largely due to incipient dementia rather than depression. J Alzheimers Dis. 2016; 54(1):185-99.

47. Mirza S, Wolters F, Swanson S, et al. 10-year trajectories of depressive symptoms and risk of dementia: a population-based study. Lancet Psychiatry. 2016;3(7):628-35.

48. Barca L, Engedal K, Laks J, Selbaek G. Factors associated with a depressive disorder in Alzheimer's disease are different from those found for other dementia disorders. Dement Geriatr Cogn Dis Extra. 2012; 2(1):19-28.

49. Weightman J, Aire T, Baune B. A review of the role of social cognition in major depressive disorder. Psiquiatría Delantera. 2014; 5:179.

50. Villarreal A, Grajales S, López L, Britton G, Panama aging resseach initiative. cognitive impairment, depression and concurrence of both among the elderly in Panama: Differential associations with multiborbidity and functional limitations. Biomed Res Int. 2015; 2015:0-0. doi: http://dx.doi.org/10.1155/2015/718701 
51. Hiltunen H, Tan E, Ilomäki J, et al. Factors associated with antidepressant use in residents with and without dementia in Australian aged facilities. Ther Adv Drug Saf. 2016; 7(3): 79-88.

52. Pigeon $\mathrm{W}$, Hegel $\mathrm{M}$, Unützer $\mathrm{J}$, et al. Is insomnia a perpetuating factor for late-life depression in the IMPACT cohort? Sleep. 2008; 31(14): 481-488.

53. Lee E, Jin Cho H, Olmestead R, Levin M, Oxman M, Irwin M. Persistent Sleep Disturbance: A risk factor recurrent depression in community dwelling older adults. Sleep. 2013; 36(11): 1685-1691.

54. Jaussent I, Bouyer J, Ancelin M, et al. Insomnia and daytime sleepiness are risk factors for depressive symptoms in the elderly. Sleep. 2011; 34(8):11031110.

55. Hawton A, Green C, Dickens A, et al. The impact of social isolation on the health status and healthrelated quality of life of older people. Qual Life Res. 2011; 20: 57-67.

56. Nazemi L, Skoong I, Karlsson I, et al. Depression, prevalence and some risk factors in elderly nursing homes in Teheran-Iran. Iranian J Publ Health. 2013; 42(6): 559-569.

57. He G, Xie J, Zhou J, Zhong Z, Qin C, Ding S. Depression in left-behind elderly in rural China: Prevalence and associated factors. Geriatr Gerontol Int. 2016;16(5):638-43.

58. Organización Mundial de la Salud. Estadísticas Mundiales: una mina de información sobre salud pública mundial. Ginebra: Organización Mundial de la Salud.; 2014.

59. Siversten H, Bjorkdof Gh, Engedal K, Selbaeck $\mathrm{G}$, Helvick AS. Depression and quality of life in older persons: A review. Dement Geriatr Cogn Disord. 2015; 40(5-6):311-339.

60. Wilkie R, Blagojevic M, Belcher J, ChewGraham C, Lacey RJ, McBeth J. Widespread pain and depression are key modifiable risk factors associated with reduced social participation in older adults. Medicine (Baltimore). 2016;95(31):e4111. doi: 10.1097/MD.0000000000004111

61. Xu H, Qin L, Wang J, et al. A cross-sectional study on risk factors and their interactions with suicidal ideation among the elderly in rural communities of Hunan, China. BMJ Open. 2016; 6(4):1-8. Doi: http://dx.doi.org/10.1136/ bmjopen-2015-010914

62. Galli R, Hideyuki E, Bruscato N, Horta R, Pattussi $\mathrm{M}$. Active aging is associated with low prevalence of depressive symptoms among Brazilian older adults. Rev Bras Epidemiol. 2016; 19(2):307-316.

63. Hsieh N. Economic Security, Social Cohesion, and Depression Disparities in Post-Transition Societies: A Comparison of Older Adults in China and Russia. J Health Soc Behav. 2015;56(4):534-51. doi: $10.1177 / 0022146515611731$
64. Foscolou A, Tyrovolas S, Soulis G, et al. The impact of the financial crisis on lifestyle health determinants among older adults living in the Mediterranean Region: The Multinational MEDIS Study (20052015). J Prev Med Public Health. 2017; 50:1-9.

65. Reyes B, Rosero-Bixby L, Koivumaa-Honkanen H. Effects of self-rated health and self-rated economic situation on depressed mood via life satisfaction among older adults in Costa Rica. J Aging Health. 2016; 28(2): 225-243. doi: 10.1177/089826431 5589577

66. Schoevers R, Geerlings M, Deeg D, Holwerda T, Jonker C, Beekman A. Depression and excess mortality: evidence for a dose response relation in community living elderly. Int $\mathrm{J}$ Geriatr Psychiatry. 2009; 24(2):169-76.

67. Hajek A, Brettschneider C, Mallon T, Ernst A, Mamone S, Wiese B. The impact of social engagement on health-related quality of life and depressive symptoms in old age - evidence from a multicenter prospective cohort study in Germany. Resultados de salud. 2017; 15 (1): 140. doi: 10.1186/s12955-0170715-8

68. Unützer J, Schoenbaum M, Katon W, et al. Healthcare costs associated with depression in medically III fee-for-service medicare participants. J Am Geriatr Soc. 2009; 57(3):506-10.

69. Instituto Nacional de Estadísticas y Censos. Salud, Bienestar y Envejecimiento. Quito: Instituto Nacional de Estadísticas y Censos; 2009. p. 17-18.

70. Lin EH, Katon W, Von Korff M, et al. Effect of improving depression care on pain and functional outcomes among older adults with arthritis: a randomized controlled trial. JAMA. 2003; 290 (18):2428-9.

71. Williams JW, Katon W, Lin EH, et al. The effectiveness of depression care management on diabetes-related outcomes in older patients. Ann Intern Med. 2004; 140(12):1015-24.

72. Choi NG, Kim J, Marti CN, Chen GJ. Latelife depression and cardiovascular disease burden: examination of reciprocal relationship. Am J Geriatr Psychiatry. 2014; 22(12):1522-9.

73. Aguilar-Navarro S, Ávila-Funes J. La depresión: particularidades clínicas y consecuencias en el adulto mayor. Gac Med Mex. 2007; 143(2):141-148.

74. Allers K, Hoffmann F. Mortality and hospitalization at the end of life in newly admitted nursing home residents with and without dementia. Soc Psychiatry Psychiatr Epidemiol. 2018;53(8):833-839. doi: 10.1007/s00127-018-1523-0

75. Georgakis MK, Papadopoulos FC, Protogerou AD, Pagonari I, Sarigianni F, Biniaris-Georgallis S. Comorbidity of cognitive impairment and late-life depression increase mortality: results from a cohort of community-dwelling elderly individuals in rural 
Greece. J Geriatr Psychiatry Neurol. 2016; 29(4):195204.

76. Luna-Matos M, Mcgrath H, Gaviria M. Manifestaciones neuropsiquiátricas en accidentes cerebrovasculares. Neuropsychiatric manifestations in cerebrovascular diseases. Rev Chil Neuro-psiquiat. 2007;45(2):129-140.

77. Damián J, Pastor-Barriuso R, Valderrama-Gama E, De Pedro-Cuesta J. Association of detected depression and undetected depressive symptoms with long-term mortality in a cohort of institutionalized older people. Epidemiol Psychiatr Sci. 2017;26(2):189-198. doi: 10.1017/S204579601500 1171

78. Almeida OP, Hankey GJ, Yeap BB, Golledge J, Flicker L. Depression as a modifiable factor to decrease the risk of dementia. Transl Psychiatry. 2017;7(5):e1117. doi: 10.1038/tp.2017.90

79. Hashem AH, Nashreldin M, Gomaa MA, Khalaf O. Late versus early onset depression in elderly patients: vascular risk and cognitive impairment. Curr Aging Sci. 2017;10(3):211-216. doi: 10.2174/18746098106 66170404105634.

80. Bolton JM, Robinson J, Sareen J. Self-medication of mood disorders with alcohol and drugs in the National Epidemiologic Survey on Alcohol and Related Conditions. J Affect Disord. 2009 115(3):36775.

81. Gum AM, King-Kallimanis B, Kohn R. Prevalence of mood, anxiety, and substance-abuse disorders for older Americans in the national comorbidity surveyreplication. Am J Geriatr Psychiatry. 2009; 17(9):76981.
82. Valenstein M, Taylor KK, Austin K, Kales HC, McCarthy JF, Blow FC. Benzodiazepine use among depressed patients treated in mental health settings. Am J Psychiatry. 2004; 161(4):654-61.

83. Blow FC, Brockmann LM, Barry KL. Role of alcohol in late-life suicide. Alcohol Clin Exp Res. 2004; 28(5 Suppl):48S-56S.

84. Waern M, Runeson BS, Allebeck P, et al. Mental disorder in elderly suicides: a case-control study. Am J Psychiatry. 2002; 159(3):450-5.

85. Sistema Nacional de Salud España. Guía de práctica clínica de prevención y tratamiento de la conducta suicida. Madrid: Sistema Nacional de Salud España; 2012.p. 218-221.

86. Raue PJ, Ghesquiere AR, Bruce ML. Suicide risk in primary care: identification and management in older adults. Curr Psychiatry Rep. 2014; 16(9):466.

87. Lapierre S, Erlangsen A, Waern M, et al. A systematic review of elderly suicide prevention programs. Crisis. 2011; 32(2):88-98.

88. Ribot V, Romero M, Ramos M, González A. Suicidio en el adulto mayor. Rev Haban Cienc Méd. 2012; 11 (supl5):699-708.

Recibido: 09/08/2018

Aceptado: 28/08/2018 